\title{
Resolução comentada da P1 de 2017 de Transferência de Calor (PME3360)
}

\author{
Éber Saj Porcacchia
}

\begin{abstract}
Resumo - A disciplina de Transferência de Calor (PME3360) costuma ser vista pelos alunos como um dos grandes desafios a serem vencidos durante a graduação da engenharia. Por associar conceitos introduzidos pela Termodinâmica à complexidade da Mecânica dos Fluidos, a matéria, de fato, demanda dos alunos habilidades de interpretação e modelagem de problemas juntamente com a manipulação algébrica. Tendo em vista tal situação, esse texto busca levar até os alunos, por meio da resolução de exercícios de prova, um material para auxiliá-los nos estudos da disciplina. Com isso, espera-se que os alunos consigam ter maior familiaridade com a aplicação da teoria aprendida em problemas reais e entendam melhor quais habilidades serão exigidas no momento da prova.
\end{abstract}

Palavras-chave - Transferência de calor; Prova; Resolução; PME3360

Title - Commented solution of P1 from 2017 of Heat Transfer (PME3360)

Abstract - The Heat Transfer course (PME3360) is often seen by students as one of the great challenges to be overcome during the engineering graduation. By associating concepts introduced by Thermodynamics with the complexity of Fluid Mechanics, the subject, in fact, demands from the students skills in problem interpretation and modeling along with algebraic manipulation. Having this in mind, this text seeks to bring to the students a material to help in the studies of the course, through the solution of test exercises. With this, the students are expected to be more familiar with applying learned theory to real world problems and to better understand what skills will be required at the time of the exam.

Keywords - Heat transfer, exam, solution, PME3360

Éber Saj Porcacchia, cursa Engenharia Mecatrônica desde 2015 e se tornou membro do PET Mecatrônica em 2017.

Versão inicial submetida em 16 de novembro de 2017. Versão final aceita em 16 de novembro de 2017. Publicado em 16 de novembro de 2017. Digital Object Identifier 10.11606/issn.2526-8260.mecatrone.2017.140641 


\section{Questão 1}

Uma haste com comprimento $2 L$, perímetro $P$ e área da seção transversal $A_{c}$ gera energia térmica a uma taxa volumétrica $q^{\prime \prime \prime}$. Metade da haste está isolada enquanto a outra transfere calor por convecção com o fluido a $T_{\infty}$. O coeficiente de transferência de calor associado é $h$. A base e a ponta da haste estão isoladas termicamente. Considerando regime permanente, pede-se para:

(a) deduzir as equações diferenciais que descrevem o perfil de temperatura em cada uma das partes.

(b) apresentar as condições de contorno.

(c) determinar o perfil de temperatura em cada parte.

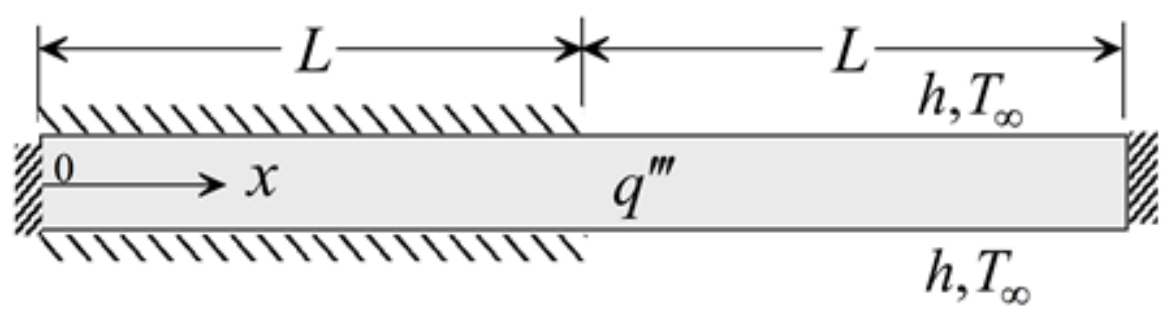

Figura 1 - Desenho do problema descrito na questão 1.

\section{Resolução}

Hipóteses

A haste está em regime permanente.

Condução unidimensional em x.

Os efeitos de radição são desprezíveis.

As propriedades do material são uniformes e constantes.

Nesse problema, temos que deduzir as expressões dos perfis de temperatura em duas regiões de uma aleta sob diferentes condições. Tomando um elemento diferencial na região da esquerda, temos o seguinte balanço de energia:

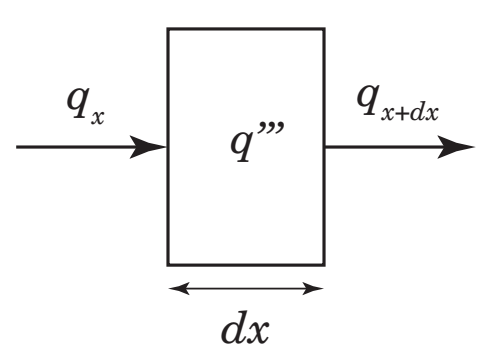

$$
\begin{gathered}
q_{x}-q_{x+\mathrm{d} x}+q^{\prime \prime \prime} \mathrm{d} x A_{c}=0 \\
q_{x}-\left(q_{x}+\frac{\mathrm{d} q_{x}}{\mathrm{~d} x} \mathrm{~d} x\right)+q^{\prime \prime \prime} \mathrm{d} x A_{c}=0 \\
-\frac{\mathrm{d} q_{x}}{\mathrm{~d} x} \mathrm{~d} x+q^{\prime \prime \prime} \mathrm{d} x A_{c}=0 \\
-\frac{\mathrm{d} q_{x}}{\mathrm{~d} x}+q^{\prime \prime \prime} A_{c}=0
\end{gathered}
$$


Pela lei de Fourier:

$$
\begin{gathered}
q_{x}=-k A_{c} \frac{\mathrm{d} T_{1}}{\mathrm{~d} x} \Longrightarrow k A_{c} \frac{\mathrm{d}^{2} T_{1}}{\mathrm{~d} x^{2}}+q^{\prime \prime \prime} A_{c}=0 \\
\frac{\mathrm{d}^{2} T_{1}}{\mathrm{~d} x^{2}}+\frac{q^{\prime \prime \prime}}{k}=0 \\
T_{1}(x)=\frac{-q^{\prime \prime \prime} x^{2}}{2 k}+C_{1} x+C_{2}
\end{gathered}
$$

Tomando um elemento diferencial na região da direita, temos o seguinte balanço de energia:

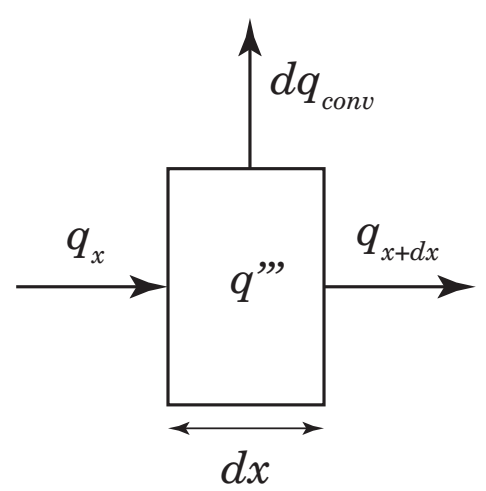

$$
\begin{gathered}
q_{x}-q_{x+\mathrm{d} x}-d q_{c o n v}+q^{\prime \prime \prime} \mathrm{d} x A_{c}=0 \\
q_{x}-\left(q_{x}+\frac{\mathrm{d} q_{x}}{\mathrm{~d} x} \mathrm{~d} x\right)-h d A_{s}\left(T_{2}-T_{\infty}\right)+q^{\prime \prime \prime} \mathrm{d} x A_{c}=0 \\
-\frac{\mathrm{d} q_{x}}{\mathrm{~d} x} \mathrm{~d} x-h P \mathrm{~d} x\left(T_{2}-T_{\infty}\right)+q^{\prime \prime \prime} \mathrm{d} x A_{c}=0 \\
-\frac{\mathrm{d} q_{x}}{\mathrm{~d} x}-h P\left(T_{2}-T_{\infty}\right)+q^{\prime \prime \prime} A_{c}=0
\end{gathered}
$$

Pela lei de Fourier:

$$
\begin{gathered}
q_{x}=-k A_{c} \frac{\mathrm{d} T_{2}}{\mathrm{~d} x} \Longrightarrow k A_{c} \frac{\mathrm{d}^{2} T_{2}}{\mathrm{~d} x^{2}}-h P\left(T_{2}-T_{\infty}\right)+q^{\prime \prime \prime} A_{c}=0 \\
\frac{\mathrm{d}^{2} T_{2}}{\mathrm{~d} x^{2}}-\frac{h P}{k A_{c}}\left(T_{2}-T_{\infty}\right)+\frac{q^{\prime \prime \prime}}{k}=0
\end{gathered}
$$

Definindo

$$
m^{2}=\frac{h P}{k A_{c}} \quad \theta_{2}(x)=T_{2}(x)-T_{\infty}
$$

Temos:

$$
\begin{gathered}
\frac{\mathrm{d}^{2} \theta_{2}}{\mathrm{~d} x^{2}}-m^{2} \theta_{2}=-\frac{q^{\prime \prime \prime}}{k} \\
\theta_{2}(x)=C_{3} e^{m x}+C_{4} e^{-m x}+\frac{q^{\prime \prime \prime} A_{c}}{h P}
\end{gathered}
$$




$$
T_{2}(x)=C_{3} e^{m x}+C_{4} e^{-m x}+\frac{q^{\prime \prime \prime} A_{c}}{h P}+T_{\infty}
$$

Agora, utilizamos as seguintes condições de contorno para determinarmos as constantes $C_{1}$, $C_{2}, C_{3}, C_{4}$.

$$
\left.\frac{\mathrm{d} T_{1}}{\mathrm{~d} x}\right|_{x=0}=0 \quad T_{1}(L)=\left.T_{2}(L) \quad \frac{\mathrm{d} T_{1}}{\mathrm{~d} x}\right|_{x=L}=\left.\left.\frac{\mathrm{d} T_{2}}{\mathrm{~d} x}\right|_{x=L} \quad \frac{\mathrm{d} T_{2}}{\mathrm{~d} x}\right|_{x=2 L}=0
$$

$$
\begin{gathered}
\frac{\mathrm{d} T_{1}}{\mathrm{~d} x}=\frac{-q^{\prime \prime \prime} x}{k}+C_{1} \\
\frac{\mathrm{d} T_{2}}{\mathrm{~d} x}=m C_{3} e^{m x}-m C_{4} e^{-m x}
\end{gathered}
$$

(I) $\Longrightarrow \frac{-q^{\prime \prime \prime} \cdot 0}{k}+C_{1}=0 \Longrightarrow C_{1}=0$

$(\mathrm{IV}) \Longrightarrow m C_{3} e^{m 2 L}-m C_{4} e^{-m 2 L}=0 \Longrightarrow m C_{3}-m C_{4}=0 \Longrightarrow C_{3} e^{4 m L}=C_{4}$

(III) $\Longrightarrow \frac{-q^{\prime \prime \prime} L}{k}+C_{1}=m C_{3} e^{m L}-m C_{4} e^{-m L} \Longrightarrow \frac{-q^{\prime \prime \prime} L}{k}=m C_{3} e^{m L}-m C_{3} e^{4 m L} e^{-m L} \Longrightarrow$ $\Longrightarrow \frac{-q^{\prime \prime \prime} L}{k}=m C_{3} e^{m L}-m C_{3} e^{3 m L} \Longrightarrow C_{3}=\frac{-q^{\prime \prime \prime} L}{m k\left(e^{m L}-e^{3 m L}\right)}$ $\therefore \quad C_{4}=C_{3} e^{4 m L}=\frac{-q^{\prime \prime \prime} L e^{4 m L}}{m k\left(e^{m L}-e^{3 m L}\right)}$

$$
\begin{aligned}
(\mathrm{II}) & \Longrightarrow \frac{-q^{\prime \prime \prime} L^{2}}{2 k}+C_{1} L+C_{2}=C_{3} e^{m L}+C_{4} e^{-m L}+\frac{q^{\prime \prime \prime} A_{c}}{h P}+T_{\infty} \Longrightarrow \\
& \Longrightarrow \frac{-q^{\prime \prime \prime} L^{2}}{2 k}+C_{2}=C_{3} e^{m L}+C_{3} e^{4 m L} e^{-m L}+\frac{q^{\prime \prime \prime} A_{c}}{h P}+T_{\infty} \Longrightarrow \\
& \Longrightarrow \frac{-q^{\prime \prime \prime} L^{2}}{2 k}+C_{2}=\frac{-q^{\prime \prime \prime} L e^{m L}}{m k\left(e^{m L}-e^{3 m L}\right)}+\frac{-q^{\prime \prime \prime} L e^{3 m L}}{m k\left(e^{m L}-e^{3 m L}\right)}+\frac{q^{\prime \prime \prime} A_{c}}{h P}+T_{\infty} \Longrightarrow \\
& \Longrightarrow C_{2}=\frac{-q^{\prime \prime \prime} L}{m k}\left(\frac{e^{m L}+e^{3 m L}}{e^{m L}-e^{3 m L}}\right)+q^{\prime \prime \prime}\left(\frac{A_{c}}{h P}+\frac{L^{2}}{2 k}\right)+T_{\infty}
\end{aligned}
$$


Portanto, os perfis de temperatura em cada parte são dados por:

$$
\begin{gathered}
T_{1}(x)=\frac{-q^{\prime \prime \prime} x^{2}}{2 k}-\frac{q^{\prime \prime \prime} L}{m k}\left(\frac{e^{m L}+e^{3 m L}}{e^{m L}-e^{3 m L}}\right)+q^{\prime \prime \prime}\left(\frac{A_{c}}{h P}+\frac{L^{2}}{2 k}\right)+T_{\infty} \\
T_{2}(x)=\frac{-q^{\prime \prime \prime} L}{m k\left(e^{m L}-e^{3 m L}\right)}\left(e^{m x}+e^{4 m L} e^{-m x}\right)+\frac{q^{\prime \prime \prime} A_{c}}{h P}+T_{\infty}
\end{gathered}
$$




\section{Questão 2}

Esferas de rolamento (aço: $\rho=7878 \mathrm{~kg} / \mathrm{m}^{3}, c_{p}=480 \mathrm{~J} / \mathrm{kg} . \mathrm{K}, k=14,2 \mathrm{~W} / \mathrm{m} . \mathrm{K}$ ) inicialmente a $20^{\circ} \mathrm{C}$ devem passar por um tratamento térmico em que serão aquecidas a pelo menos $550^{\circ} \mathrm{C}$. $\mathrm{O}$ aquecimento será executado pela passagem das esferas por um líquido em ebulição a $600^{\circ} \mathrm{C}$ $\left(h>50000 \mathrm{~W} / \mathrm{m}^{2} \mathrm{~K}\right)$ através de uma esteira rolante com $3 \mathrm{~m}$ de comprimento. A velocidade mínima da esteira é de $1 \mathrm{~cm} / \mathrm{s}$. As menores esferas a serem tratadas têm raio de $50 \mathrm{~mm}$. Determine qual o maior raio de esfera que pode ser tratada por esse processo.

\section{Resolução}

Dados do enunciado.

Massa específica do aço $\rho=7878 \mathrm{~kg} / \mathrm{m}^{3}$

Calor específico do aço $c_{p}=480 \mathrm{~J} / \mathrm{kg} . \mathrm{K}$

Condutividade térmica do aço $k=14,2 \mathrm{~W} / \mathrm{m} . \mathrm{K}$

Esfera com raio $r_{o} \geqslant 50 \mathrm{~mm}=0,05 \mathrm{~m}$

Temperatura inicial $T_{i}=20^{\circ} \mathrm{C}$

Temperatura final de no mínimo $T_{f}=550^{\circ} \mathrm{C}$

Convecção com $T_{\infty}=600^{\circ} \mathrm{C}$ e $h>50000 \mathrm{~W} / \mathrm{m}^{2} \mathrm{~K}$

Esteira de comprimento $l=3 \mathrm{~m}$ e velocidade $v \geqslant 1 \mathrm{~cm} / \mathrm{s}=0,01 \mathrm{~m} / \mathrm{s}$

\section{Hipóteses}

Temperatura inicial uniforme em toda a esfera.

Condução unidimensional na direção radial.

Propriedades são uniformes e constantes ao longo do processo.

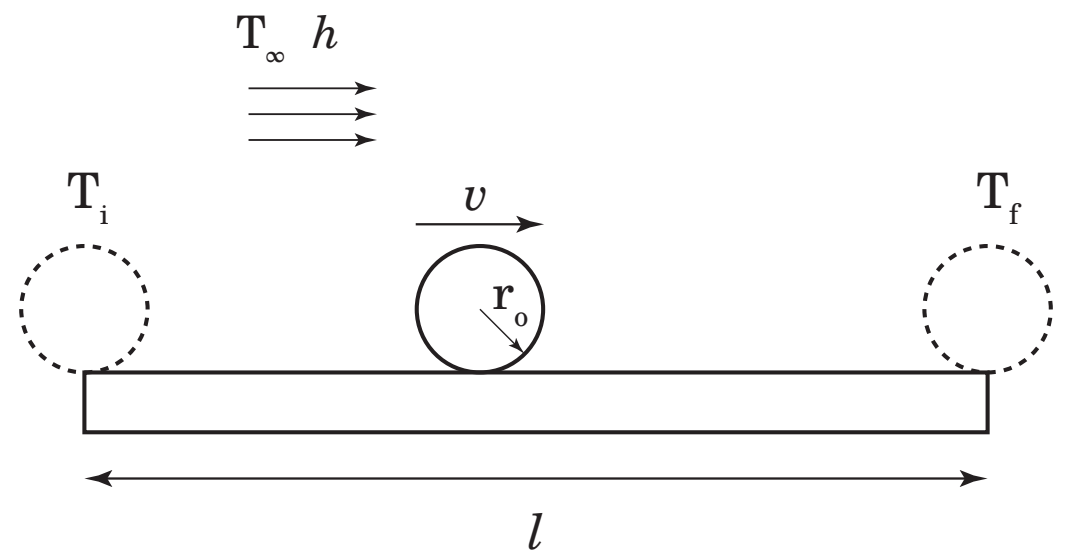

Figura 2 - Esquema do problema descrito na questão 2. 
Esse exercício envolve o aquecimento de um corpo esférico ao longo do tempo, caracterizando um problema de condução transiente. Primeiramente, vamos calcular o número de Biot para determinar se o método da capacitância global pode ser aplicado ou não. Para uma esfera, o número de Biot desse teste é dado por:

$$
\begin{gathered}
B i=\frac{h L_{c}}{k}=\frac{h r_{o}}{3 k} \\
h>50000, r_{o}>0,05 \Longrightarrow B i>\frac{50000 \cdot 0,05}{3 \cdot 14,2}=58,7
\end{gathered}
$$

Como $B i>0,1$ o método da capacitância global não é válido. Logo, devemos levar em conta os efeitos espaciais nos cálculos do problema. Para tanto, devemos calcular o número de Fourier e verificar se as soluções aproximadas são válidas.

$$
F_{O}=\frac{\alpha t}{r_{o}^{2}}
$$

Cálculo da difusividade térmica $\alpha$ do aço:

$$
\alpha=\frac{k}{\rho c_{p}}=\frac{14,2}{7878 \cdot 480}=3,75 \cdot 10^{-6} \mathrm{~m}^{2} / \mathrm{s}
$$

Precisamos calcular também o tempo $t$ durante o qual a esfera será aquecida. Sabemos que a esteira tem um comprimento $l=3 \mathrm{~m}$ e caminha com uma velocidade $v=0,01 \mathrm{~m} / \mathrm{s}$, portanto o tempo é dado por:

$$
t=\frac{l}{v}=\frac{3}{0,01}=300 \mathrm{~s}
$$

Com isso, o número de Fourier é dado por:

$$
F_{O}=\frac{\alpha t}{r_{o}^{2}}=\frac{3,75 \cdot 10^{-6} \cdot 300}{0,05^{2}}=0,45
$$

Como $F_{O}>$ 0,2 podemos utilizar a aproximação pelo primeiro termo da série. No caso da esfera, é dada por:

$$
\begin{gathered}
\theta^{*}=\theta_{o}^{*} \frac{1}{\zeta_{1} r^{*}} \operatorname{sen}\left(\zeta_{1} r^{*}\right), \text { onde } \theta^{*}=\frac{T(r, t)-T_{\infty}}{T(r, 0)-T_{\infty}} \\
\theta_{o}^{*}=C_{1} \exp \left(-\zeta_{1}^{2} F_{o}\right)
\end{gathered}
$$


Onde $\theta_{o}^{*}$ representa a temperatura no centro da esfera, ou seja:

$$
\theta_{o}^{*}=\frac{T(0, t)-T_{\infty}}{T(r, 0)-T_{\infty}}
$$

O próximo passo é determinar os coeficientes $C_{1}$ e $\zeta_{1}$. Para isso, utilizaremos a tabela 5.1 do livro-texto (INCROPERA et al., 2008), que possui como entradas a geometria do problema (parede plana, cilindro infinito ou esfera) e o número de Biot, agora definido como:

$$
B i=\frac{h r_{o}}{k}>\frac{50000 \cdot 0,05}{14,2}=176
$$

Tabela 1 - Coeficientes usados na aproximação pelo primeiro termo das soluções em série da condução unidimensional transiente.

\begin{tabular}{|c|cc|cc|cc|}
\hline & \multicolumn{2}{|c|}{ Parede Plana } & \multicolumn{2}{|c|}{ Cilindro Infinito } & \multicolumn{2}{c|}{ Esfera } \\
\hline $\mathrm{Bi}$ & $\zeta_{1}$ & $C_{1}$ & $\zeta_{1}$ & $C_{1}$ & $\zeta_{1}$ & $C_{1}$ \\
\hline 50,0 & 1,5400 & 1,2727 & 2,3572 & 1,6002 & 3,0788 & 1,9962 \\
100,0 & 1,5552 & 1,2731 & 1,6015 & 1,6015 & 3,1102 & 1,9990 \\
$\infty$ & 1,5708 & 1,2733 & 1,6018 & 1,6018 & 3,1415 & 2,0000 \\
\hline
\end{tabular}

Como a tabela possui valores apenas até $B i=100$, pegaremos os valores de $C_{1}$ e $\zeta_{1}$ na linha $B i=\infty$.

$$
\zeta_{1}=3,1415 \text { e } C_{1}=2,0000
$$

Queremos que o centro da esfera, que inicialmente está à temperatura $T_{i}=20^{\circ} \mathrm{C}$, esteja à temperatura $T_{f}=550^{\circ} \mathrm{C}$ após $t=300 \mathrm{~s}$. Portanto, temos que resolver a seguinte equação para descobrirmos o raio máximo $R$.

Hipótese: supomos aqui que a solução aproximada continuará sendo válida para um esfera de raio máximo $r_{o}=R$, ou seja, ainda teremos $F_{O}>0,2$. A validade dessa hipótese terá que ser verificada ao final do exercício.

$$
\begin{gathered}
\theta_{o}^{*}=\frac{T(0, t)-T_{\infty}}{T(r, 0)-T_{\infty}}=C_{1} \exp \left(-\zeta_{1}^{2} F_{o}\right) \\
\frac{T_{f}-T_{\infty}}{T_{i}-T_{\infty}}=C_{1} \exp \left(-\zeta_{1}^{2} \frac{\alpha t}{R^{2}}\right) \\
\frac{550-600}{20-600}=2 \exp \left[-(3,1415)^{2} \frac{3,75 \cdot 10^{-6} \cdot 300}{R^{2}}\right] \\
R=0,0594 \mathrm{~m}=59,4 \mathrm{~mm}
\end{gathered}
$$


Cálculo do número de Fourier para um esfera de raio máximo $R$ :

$$
F_{O}=\frac{\alpha t}{R^{2}}=\frac{3,75 \cdot 10^{-6} \cdot 300}{0,0594^{2}}=0,32
$$

Cálculo do novo número de Biot:

$$
B i=\frac{h R}{k}=\frac{50000 \cdot 0,0594}{14,2}=209
$$

Como Fo continua maior que 0,2 nossa hipótese é válida e, portanto, chegamos ao raio máximo pedido pelo enunciado de $R=59,4 \mathrm{~mm}$.

\section{REFERÊNCIAS}

INCROPERA, Frank P.; DEWITT, David P.; BERGMAN, Theodore L.; LAVINE, Adrienne S.. Fundamentos de Transferência de Calor e de Massa. 6ª Edição, Rio de Janeiro: LTC, 2008. 